Classification

Physics Abstracts

$7.20 \mathrm{~K}-7.60 \mathrm{D}-44.40-44.50$

\title{
Techniques de mesure des caractéristiques radiatives des matériaux opaques
}

\author{
Ph. Demont (*), M. Huetz-Aubert ( $\left.{ }^{*}\right)$ et J. F. Sacadura $\left({ }^{* *}\right)$ \\ (*) Groupe de Recherches Thermiques du C.N.R.S. associé à l'Ecole Centrale des Arts et Manufactures, \\ Grande Voie des Vignes, 92230 Châtenay Malabry, France \\ (**) Laboratoire d'Hydraulique et Dynamique des Gaz, Institut National des Sciences Appliquées de Lyon, \\ Bât. 302, 20, avenue Einstein, 69621 Villeurbanne Cedex, France
}

(Reçu le 6 octobre 1981, révisé le 7 décembre 1981, accepté le 15 décembre 1981)

\begin{abstract}
Résumé. - Cet article est consacré à une revue synthétique des méthodes de mesure des propriétés radiatives des matériaux opaques. Après un rappel des différents paramètres radiatifs que l'on peut avoir à connaître et des relations permettant la détermination indirecte de certains d'entre eux, on donne une description critique et comparative de leurs principales techniques d'obtention. La démarche adoptée commence par l'examen des méthodes de mesure du paramètre radiatif le plus fin et peut-être le plus délicat à mesurer - la réflectivité bidirectionnelle monochromatique - et s'achève sur les paramètres les plus grossiers, à savoir les facteurs totaux hémisphériques, en passant par les différents facteurs intégrés sur l'espace ou la longueur d'onde. Un accent particulier est mis sur certaines causes d'erreur communes à la plupart des montages décrits, en particulier celles dues aux phénomènes de polarisation. Un moyen de s'en affranchir est indiqué.

Abstract. - This paper reviews the experimental techniques used in measuring the thermal radiation properties of opaque materials. After a survey of the different radiation parameters and the relations allowing reciprocal determination of some of them, a critical description of the main measurement techniques is made. The review extends from examining the methods of measuring spectral bidirectional reflectance, from which all other radiation parameters can be theoretically derived, to methods used for total hemispherical parameters. An emphasis is made on experimental error problems, specially those involved with polarization phenomena. Some means of avoiding these errors are suggested.
\end{abstract}

1. Introduction. - La mesure des températures sans contact (pyrométrie "optique») ou le calcul des échanges thermiques radiatifs sont des domaines dans lesquels la connaissance des propriétés radiatives des matériaux concernés conditionne la réussite de toute entreprise. Lorsqu'il s'agit de matériaux opaques $\left({ }^{1}\right)$, et c'est très souvent le cas dans la pratique, le rayonnement peut être considéré comme un phénomène surfacique et il s'agit alors de connaître les émissivités, absorptivités et réflectivités des surfaces en présence.

Dans la plupart des applications techniques courantes, on se contente d'évaluations approchées des transferts radiatifs utilisant les hypothèses simplificatrices usuelles :

- émission et absorption grises (on entend par là que ces propriétés ne varient pratiquement pas avec la longueur d'onde),

( $\left.{ }^{1}\right)$ On n'abordera pas dans cet article les méthodes d'étude des propriétés radiatives des milieux semi-transparents.
- émission diffuse-isotrope (l'émissivité est alors la même dans toutes les directions),

- réflexion diffuse-isotrope ou spéculaire. (La réflexion est dite diffuse-isotrope lorsque la luminance du rayonnement réfléchi est constante dans toutes les directions et ne dépend pas de la direction d'incidence. Dans la réflexion spéculaire, les directions incidente et réfléchie sont coplanaires et symétriques par rapport à la normale à la surface du point d'incidence.)

Toutefois, dans certains domaines de technologie avancée (aérospatial, énergétique nucléaire ou solaire concentrée), les impératifs de sécurité ou d'économie nécessitent que l'on prenne en compte, dans la modélisation thermique, les propriétés radiatives des matériaux dans leurs conditions réelles d'utilisation. Cela signifie qu'il faut tenir compte de leur variation avec la longueur d'onde, la direction, la température, l'état de surface, etc. Sur le plan du calcul, la chose est devenue possible grâce au développement, au cours de la dernière décennie, de méthodes numériques puissantes, par exemple les méthodes de 
Monte-Carlo qui permettent de venir à bout de problèmes très complexes de transferts radiatifs [1-3].

Mais, de graves difficultés subsistent au niveau de la connaissance des propriétés radiatives des matériaux. Il suffit d'avoir besoin, ne serait-ce qu'une fois, de rechercher des données dans des tables de manuels sur le rayonnement [1] ou même dans des recueils de données thermophysiques aussi importants que celui de Touloukian et Dewitt [4], pour prendre conscience de l'ampleur du problème : la dispersion des valeurs de la littérature et le manque d'informations précises quant à la caractérisation des matériaux faisant l'objet de compilations rendent souvent cette recherche infructueuse et obligent l'intéressé à recourir à la mesure des facteurs cherchés.

2. Les paramètres à mesurer. - Quels sont les paramètres que l'on peut avoir à connaître? En principe, il suffirait d'un seul puisque tous les autres s'en déduisent. Considérons en effet la réflectivité monochromatique bidirectionnelle $\left(^{2}\right) \rho_{\lambda}^{\prime \prime}\left(\theta, \varphi ; \theta_{\mathrm{r}}, \varphi_{\mathrm{r}}\right)$, relative à la direction d'incidence $\Delta(\theta, \varphi)$ et à la direction de réflexion $\Delta_{\mathrm{r}}\left(\theta_{\mathrm{r}}, \varphi_{\mathrm{r}}\right)($ Fig. 1). Si l'on adopte

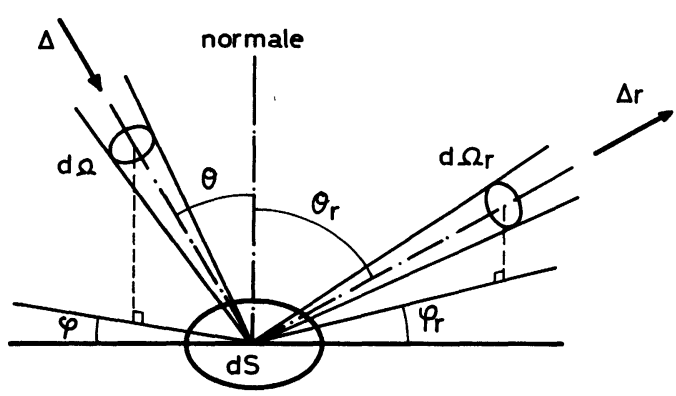

Fig. 1. - Repérage des directions d'incidence et de réflexion.

[Incidence and reflection directions.]

la définition de la référence [1], $\rho_{\lambda}^{\prime \prime}$ est le rapport de la luminance du flux réfléchi selon $\Delta_{\mathrm{r}}$ au flux surfacique incident (ou éclairement) $\mathrm{d} \phi_{\lambda \mathrm{i}}$ (de luminance $L_{\lambda \mathrm{i}}$ ) selon la direction $\Delta$ dans l'angle solide élémentaire $\mathrm{d} \Omega$. Les deux facteurs de réflexion les plus couramment utilisés s'expriment à partir de $\rho_{\lambda}^{\prime \prime}$. C'est ainsi que :

2.1 LA RÉFLECTIVITÉ MONOCHROMATIQUE DIRECTIONNELLE-HÉMISPHÉRIQUE

$$
\rho_{\lambda}^{\prime O}(\theta, \varphi)=\int_{0} \rho_{\lambda}^{\prime \prime}\left(\theta, \varphi ; \theta_{\mathrm{r}}, \varphi_{\mathrm{r}}\right) \cos \theta_{\mathrm{r}} \mathrm{d} \Omega_{\mathrm{r}}
$$

représente le rapport entre le flux surfacique réfléchi dans tout le demi-espace et $\mathrm{d} \phi_{\lambda \mathrm{i}}$; le symbole $\square$ désigne l'angle solide hémisphérique entourant l'élément de surface $\mathrm{d} S ; \mathrm{d} \Omega_{\mathrm{r}}$ est un angle solide élémentaire entourant la direction $\Delta_{\mathrm{r}}\left(\theta_{\mathrm{r}}, \varphi_{\mathrm{r}}\right)$.

$\left({ }^{2}\right)$ Notations : le symbole caractérise les grandeurs directionnelles, le symbole " les grandeurs bidirectionnelles et $\triangle$ les grandeurs hémisphériques.
2.2 LA RÉFLECTIVITÉ MONOCHROMATIQUE HÉMISPHÉRIQUE-DIRECTIONNELLE est définie comme le rapport de la luminance du flux réfléchi dans la direction $\Delta_{r}$ à la luminance moyenne du flux incident, soit :

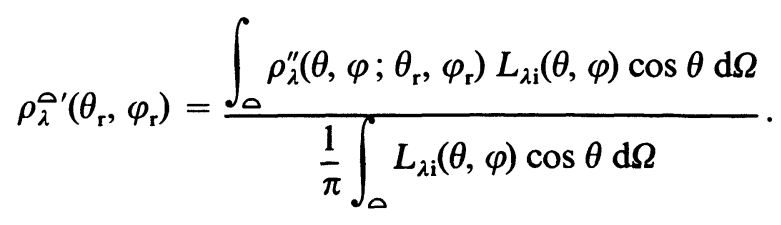

$\mathrm{d} \Omega$ est ici l'angle solide élémentaire autour de la direction $\Delta(\theta, \varphi)$.

L'expression (2) se simplifie si le flux incident est isotrope, la luminance $L_{\lambda \mathrm{i}}$ devenant indépendante de la direction $\Delta$; nous adopterons alors la notation $\rho_{\lambda \text { iso }}^{\mathrm{Q}}\left(\theta_{\mathrm{r}}, \varphi_{\mathrm{r}}\right)$ avec :

$$
\rho_{\lambda \text { iso }}^{\mathrm{O}}\left(\theta_{\mathrm{r}}, \varphi_{\mathrm{r}}\right)=\int_{0} \rho_{\lambda}^{\prime \prime}\left(\theta, \varphi ; \theta_{\mathrm{r}}, \varphi_{\mathrm{r}}\right) \cos \theta \mathrm{d} \Omega .
$$

Ces différentes relations sont détaillées dans la référence [5]. Mais, ce qu'il importe ici de remarquer, c'est que le principe de réciprocité [5] permet d'écrire :

$$
\rho_{\lambda}^{\prime \prime}\left(\theta, \varphi ; \theta_{\mathrm{r}}, \varphi_{\mathrm{r}}\right)=\rho_{\lambda}^{\prime \prime}\left(\theta_{\mathrm{r}}, \varphi_{\mathrm{r}} ; \theta, \varphi\right)
$$

de sorte que

$$
\rho_{\lambda}^{\prime \ominus}(\theta, \varphi)=\rho_{\lambda \text { iso }}^{\mathrm{O}}(\theta, \varphi)
$$

Par conséquent, tous les facteurs s'obtiennent successivement puisque, comme le rappelle l'article suivant [6], l'émissivité monochromatique directionnelle $\varepsilon_{\lambda}^{\prime}(\theta, \varphi)$ :

$$
\varepsilon_{\lambda}^{\prime}(\theta, \varphi)=1-\rho_{\lambda \text { iso }}^{\mathrm{Q}}(\theta, \varphi)
$$

devient égale à l'absorptivité monochromatique directionnelle $\alpha_{\lambda}^{\prime}(\theta, \varphi)$

$$
\alpha_{\lambda}^{\prime}(\theta, \varphi)=1-\rho_{\lambda}^{\prime \rho}(\theta, \varphi),
$$

conformément à la loi de Kirchhoff, écrite le plus souvent directement sous la forme :

$$
\varepsilon_{\lambda}^{\prime}(\theta, \varphi)=\alpha_{\lambda}^{\prime}(\theta, \varphi) .
$$

Autrement dit, la réflectivité bidirectionnelle $\rho_{\lambda}^{\prime \prime}$ indique non seulement comment un matériau réfléchit - la réalité se situant en général entre les lois spéculaire et diffuse - mais conduit également aux facteurs $\varepsilon_{\lambda}^{\prime}$ et $\alpha_{\lambda}^{\prime}$ qui interviennent dans les bilans d'énergie [7]. Malheureusement, comme nous le verrons plus loin, $\rho_{\lambda}^{\prime \prime}$ est de beaucoup le paramètre le plus difficile à mesurer. C'est pourquoi, après un paragraphe qui sera consacré à sa mesure, nous examinerons les montages plus classiques permettant d'atteindre les facteurs monochromatiques directionnels $\varepsilon_{\lambda}^{\prime}$ ou $\rho_{\lambda \text { iso }}^{\prime \prime}=\rho_{\lambda}^{\prime Q}$, puis ceux très utilisés encore à l'heure actuelle et orientés vers la détermination de facteurs, soit intégrés 
sur une bande plus ou moins large de longueur d'onde, soit hémisphériques. Auparavant, il nous semble utile d'examiner un problème qui intervient dans toutes les méthodes de mesure, à savoir l'influence de la polarisation du rayonnement, qu'il soit incident, réfléchi ou émis.

3. Les problèmes liés à la polarisation du rayonnement. - La quasi-totalité des valeurs de paramètres radiatifs rapportées dans la littérature sont issues d'expériences dans lesquelles il n'était pas tenu compte des phénomènes de polarisation du rayonnement, ce qui peut conduire à des erreurs non négligeables, ainsi que nous le montrons par ailleurs [7, 8].

En effet, le rayonnement électromagnétique pouvant être résolu en deux composantes orthogonales de polarisation, il est possible de calculer théoriquement, pour un matériau à surface idéalement plane, les deux composantes de l'émissivité : $\varepsilon_{\lambda \|}^{\prime}$, correspondant à une polarisation parallèle au plan défini par la normale à la surface et la direction d'émission et $\varepsilon_{\lambda \perp}^{\prime}$ perpendiculaire à ce plan. Ce calcul se fait à l'aide des relations de Fresnel, connaissant les indices optiques de réfraction $\eta$ et d'extinction $\kappa$ du matériau. Le tracé des indicatrices d'émissivité ainsi obtenues (Fig. 2) montre qu'en dehors des directions d'émission voisines de la
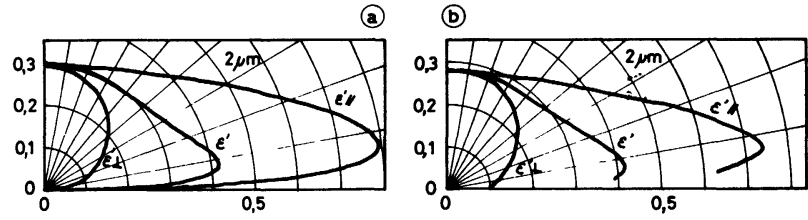

Fig. 2. - Indicatrices d'émissivité de l'acier inoxydable $304 \mathrm{~L}$ poli : a) calculées par les relations de Fresnel, à l'aide de valeurs de $\eta$ et $\kappa$ déterminées à partir de mesures de réflectivité spéculaire à $395 \mathrm{~K}[7] ; b$ ) expérimentales, obtenues indirectement par des mesures de réflectivité spéculaire à $395 \mathrm{~K}$ [7]. Le montage utilisé est du même type que celui de la figure 5 .

[Polished $304 \mathrm{~L}$ stainless steel spectral directional emittance curves (in polar coordinates) : $a$ ) calculated by Fresnel relations with optical constants, $\eta$ and $\kappa$, values derived from specular reflectance measurements performed at $395 \mathrm{~K}[7] ; b$ ) experimental, obtained indirectly from specular reflectance measurements at $395 \mathrm{~K}$. Apparatus used is of the same type as the one shown in figure 5.]

normale à la surface, pour la plupart des matériaux et surtout les métaux, $\varepsilon_{\lambda \|}^{\prime}$ présente des valeurs beaucoup plus importantes que $\varepsilon_{\lambda \perp}^{\prime}$. Dans le cas des matériaux rugueux, certains modèles théoriques ont permis de prévoir [7] et l'expérience a confirmé [7] que l'on pouvait se trouver en présence de composantes de polarisation $/ /$ et $\perp$ différentes même pour le rayonnement émis normalement à la surface (Fig. 3).

D'une manière générale, on peut donc dire que l'émission, la réflexion et la réfraction d'un rayonnement selon des directions non normales aux dioptres sont accompagnées d'une polarisation de ce rayonne-
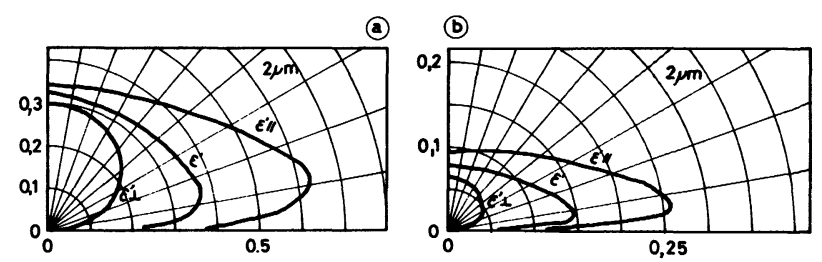

Fig. 3. - Indicatrices d'émissivité expérimentales de métaux dépolis, obtenues par mesure directe (méthode décrite au paragraphe 2) : a) acier inoxydable $304 \mathrm{~L}$ dépoli à l'abrasif No 80. Dépolissage unidirectionnel perpendiculaire au plan d'émission. Mesures à $500^{\circ} \mathrm{C}[7] ; b$ ) aluminium déposé sur un substrat en dural dépoli à l'abrasif 400. Dépolissage unidirectionnel perpendiculaire au plan d'émission. $250^{\circ} \mathrm{C}$ [7].

[Rough metals spectral directional emittance curves obtained by direct measurement (method described in 2) :a) $304 \mathrm{~L}$ stainless steel roughened using 80 grit silicon carbide paper. Monodirectional roughening normal to emission plane. Measurements performed at $\left.500{ }^{\circ} \mathrm{C}[7] ; b\right)$ vacuum deposited pure aluminum layer on a Duraluminum substrate roughened using 100 grit $\mathrm{SiC}$ paper. Monodirectional roughening normal to emission plane. $250^{\circ} \mathrm{C}$ [7].]

ment. La traversée d'un système optique toujours plus ou moins polarisant (comme le sont la plupart des dispositifs de mesure) par un rayonnement initialement plus ou moins polarisé lui-même, se traduira par des atténuations dues aux croisements éventuels des diverses polarisations.

Sans vouloir aborder les aspects théoriques du problème exposés ailleurs $[7,8]$, nous signalerons simplement qu'un moyen de s'affranchir des erreurs dues aux phénomènes de polarisation consiste à placer un polariseur sur le trajet optique et à pratiquer toutes les mesures successivement selon deux directions de polarisation perpendiculaires. Cette technique, que nous avons introduite systématiquement dans nos différents montages, permet d'atteindre la grandeur relative au rayonnement non polarisé, par exemple $\varepsilon_{\lambda}^{\prime}$, comme la moyenne arithmétique des résultats obtenus pour les deux composantes de polarisation orthogonales :

$$
\varepsilon_{\lambda}^{\prime}=\frac{1}{2}\left(\varepsilon_{\lambda \|}^{\prime}+\varepsilon_{\lambda \perp}^{\prime}\right) .
$$

Outre l'intérêt d'une exploration plus fine du phénomène, on s'affranchit ainsi d'une cause d'erreur trop souvent négligée.

4. Mesure des réflectivités bidirectionnelles. - Le schéma de principe de la mesure de la réflectivité bidirectionnelle $\rho_{\lambda}^{\prime \prime}$ est donné à la figure 4 . Ce montage nécessite un porte-échantillon à trois degrés de liberté si l'on désire mesurer la réflexion dans des directions situées en dehors du plan d'incidence [9]. Dans ce cas, il faut également disposer deux polariseurs, l'un sur le faisceau incident, lautre sur le faisceau réfléchi, compte tenu des phénomènes de dépolarisation pouvant se produire avec certains échantillons rugueux [7]. 


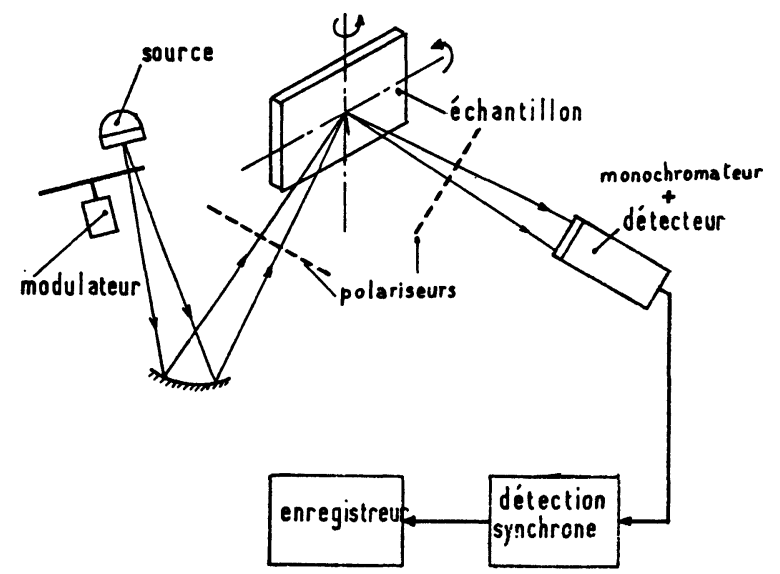

Fig. 4. - Schéma de principe d'un montage de mesure de la réflectivité bidirectionnelle.

[Schematic diagram of a bidirectional reflectance measurement facility.]

Dans la plupart des cas, on se limite cependant à l'étude de la réflexion dans le plan d'incidence [10] et le porte-échantillon peut être alors réalisé à l'aide d'un goniomètre dont le plateau porte-prismes est adapté en conséquence. La réflexion dans le plan d'incidence ne donnant pas lieu à des dépolarisations [7], un seul polariseur est alors nécessaire.

La plupart des dispositifs réalisés à ce jour, dont nous avons eu connaissance, étaient destinés à des mesures de $\rho_{\lambda}^{\prime \prime}$ sur des échantillons à la température ambiante, bien que rien ne s'oppose en principe à effectuer des mesures de $\rho_{\lambda}^{\prime \prime}$ sur un échantillon chaud : le fait de moduler le flux incident permet de séparer, par la détection synchrone, le flux réfléchi du flux émis par l'échantillon.

La figure 5 donne le schéma d'un dispositif utilisé par Lafait et Behaghel au Laboratoire d'Optique des Solides (Université Paris VI) pour la mesure de la réflectivité bidirectionnelle spéculaire. Ce montage est opérationnel dans le domaine 1,5 à $15 \mu \mathrm{m}$ [11].

Un montage permettant d'atteindre à la fois $\rho_{\lambda}^{\prime \prime}$ et $\varepsilon_{\lambda}^{\prime}$ a été mis au point par Sacadura, à l'I.N.S.A. de Lyon [7]; on trouvera sa description dans le prochain article [6].

De tels montages peuvent être en principe adaptés à la mesure de $\rho_{\lambda}^{\prime \prime}$ dans le visible en remplaçant simplement le prisme (ou le réseau) du monochromateur, la source, le détecteur et le polariseur par des éléments correspondants fonctionnant dans le domaine visible : ainsi, la source du type globar ou Nernst est remplacée par une lampe et l'on substitue au détecteur I.R. (thermopile, pyroélectrique ou quantique) une photodiode ou un photomultiplicateur. Cependant, l'utilisation d'un photomultiplicateur nécessite sa protection contre toute lumière parasite - même non modulée ce qui implique que le dispositif soit entièrement clos dans un boîtier si l'on veut éviter d'avoir à manipuler en salle obscure. De ce fait, le système représenté à la figure 4 n'a pas été réutilisé dans le domaine du visible,

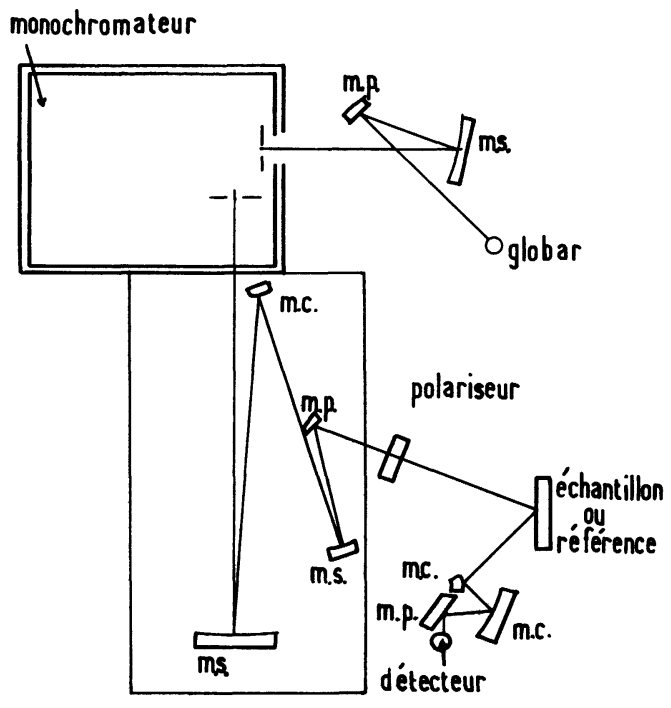

Fig. 5. - Mesure de la réflectivité $\rho_{\lambda}^{\prime S}$ (spéculaire). Montage du Laboratoire d'Optique des Solides, Université Paris VI. m.p. $=$ miroir plan $;$ m.s. $=$ miroir sphérique $;$ m.c. $=$ miroir convexe.

[Measurement of specular reflectance $\rho_{\lambda}^{\prime \mathbf{s}}$. Apparatus used by Laboratoire d'Optique des Solides, University of Paris VI. m.p. $=$ plane mirror $; \mathrm{m} . \mathrm{s}$. $=$ spherical mirror $;$ m.c. $=$ convex mirror.]

pour lequel il a paru préférable de réaliser un autre dispositif indépendant.

Les mesures de $\rho_{\lambda}^{\prime \prime}$ sont, à l'heure actuelle, surtout utilisées soit pour la détermination des indices optiques des matériaux [7, 12], soit pour l'étude de la rugosité de surface et de son influence sur la réflexion [7, 9, $10,13]$.

Une des grosses difficultés qui limite ce type de mesures est le manque d'énergie, surtout dans l'infrarouge, avec les échantillons diffusants. Cependant, l'utilisation de lasers [14] puissants à la place des traditionnels globar ou filaments de Nernst et le développement des systèmes d'acquisition de données pour l'exploration automatisée des différentes directions de l'espace devraient ouvrir de nouvelles possibilités à ce type de montage dont l'intérêt est évident, la mesure de $\rho_{\lambda}^{\prime \prime}$ permettant d'accéder en principe à tous les autres facteurs, comme nous lavons vu au paragraphe 2 .

5. Mesure directe des émissivités directionnelles. La mesure directe de $\varepsilon_{\lambda}^{\prime}$ s'effectue par comparaison des flux émis par l'échantillon et par un corps noir porté à la même température [7, 15-16]. Un système optique (Fig. 6) définit la surface observée et l'angle solide d'émission, tandis qu'un monochromateur ou un système de filtres détermine la longueur d'onde et la largeur spectrale de la mesure. Les mesures directionnelles sont facilement obtenues par rotation de l'échantillon autour d'un axe situé dans le plan de sa surface. L'utilisation d'un polariseur permet d'éviter les erreurs signalées précédemment. L'introduction 


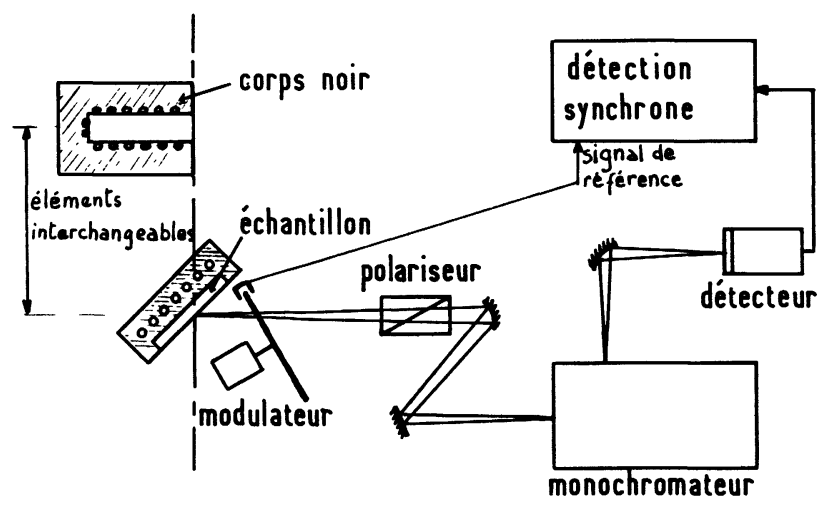

Fig. 6. - Principe de la mesure directe de l'émissivité monochromatique directionnelle.

[Principle of spectral directional emittance measurement by direct method.]

d'un modulateur de faisceau joint à un amplificateur à détection synchrone, qui ne conserve du signal que la composante de fréquence égale à celle du modulateur, élimine une bonne part des rayonnements parasites.

Le domaine d'utilisation est déterminé par la sensibilité de la chaîne de mesure (surtout du détecteur) et est limité par la nécessité d'une émission substantielle de l'échantillon, ce qui, en ordre de grandeur, n'est réalisé que pour des longueurs d'onde comprises entre 2 et $20 \mu \mathrm{m}$ et des températures supérieures à $500 \mathrm{~K}$. A plus basse température, la part non négligeable du rayonnement réfléchi à la surface de l'échantillon enlèverait du reste toute signification aux mesures, surtout dans le cas des matériaux peu émissifs comme la plupart des métaux polis. Une analyse des erreurs associées à ce type de mesures est détaillée dans le prochain article [6].

Une difficulté inhérente à cette méthode est qu'elle nécessite une détermination précise de la température de surface de l'échantillon qui doit être rigoureusement égale à celle du corps noir, ce qui n'est pas aisé pour les matériaux mauvais conducteurs de la chaleur. En effet, de faibles écarts de température - quelques degrés à peine - entre corps noir et échantillon peuvent provoquer des erreurs de mesure importantes. De ce fait, de nombreux systèmes de chauffage ont été utilisés dans le passé, en particulier pour la mesure des émissivités de corps réfractaires [17]. Par ailleurs, le schéma de la chaîne optique doit être conçu pour éviter les réflexions parasites, autrement dit qu'une partie du flux émis par l'échantillon ne soit réfléchie par le modulateur (ou tout autre élément de la chaine optique) vers l'échantillon, augmentant ainsi le flux émis d'une composante réfléchie qui n'est pas éliminée par la détection synchrone. Il convient de plus que la modulation du faisceau ait lieu le plus près possible de la source, tout en essayant de limiter l'échauffement du modulateur dont les pales émettent à leur tour un signal en opposition de phase avec celui de l'échantillon.
On notera enfin que les mesures directionnelles nécessitent un échantillon de grandes dimensions, puisque l'aire de la surface vue par le détecteur augmente au fur et à mesure que la normale à l'échantillon s'éloigne de l'axe de visée.

Des précautions particulières sont donc nécessaires pour assurer et contrôler l'isothermie de la surface concernée. Hervé a utilisé pour ce faire la photothermométrie et une sonde de température de surface [15]. Dans le prochain article [6], les auteurs décriront les solutions qu'ils ont apportées aux différents problèmes évoqués ci-dessus.

6. Mesure des réflectivités directionnelle-hémisphérique et hémisphérique-directionnelle. - L'idée de recueillir sur un détecteur ce qu'un échantillon, recevant un flux directionnel, réfléchit dans toutes les directions, est attribuée à Paschen, en 1899. C'est à peu près vers cette époque que la méthode inverse dans laquelle, à partir d'un flux incident hémisphérique, on mesure le flux réfléchi selon une direction donnée, a également été envisagée. De nombreux systèmes de mesure utilisant ces deux principes ont été proposés depuis, qui peuvent être classés en trois catégories : les systèmes à miroirs, les sphères intégrantes et les cavités chauffées.

6.1 Utilisation Des SYSTÈMES À MIROIRS. - Pour atteindre $\rho^{\prime}$, , Coblentz [18] en 1913 a proposé d'utiliser une demi-sphère en verre poli, recouverte d'un métal très réfléchissant et dans laquelle l'échantillon et un détecteur sont disposés, symétriquement par rapport au centre (Fig. 7). Le flux incident rentre dans l'hémisphère par un orifice et, après réflexion sur l'échantillon, puis le miroir sphérique, revient sur le détecteur. Par substitution d'une référence à l'échantillon, on détermine $\rho^{\prime \iota}$.

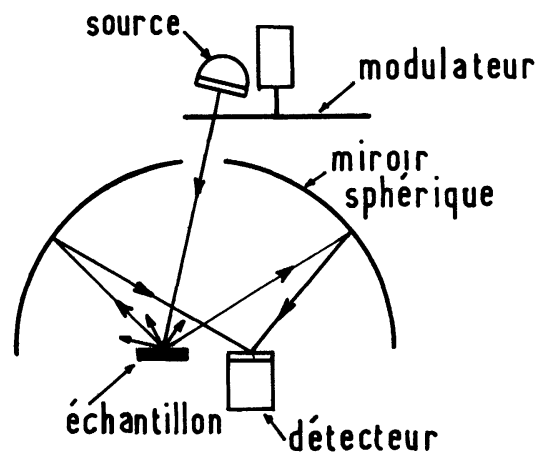

Fig. 7. - Miroir hémisphérique de Coblentz $\left(\rho^{\prime 0}\right)$.

[Coblentz's hemispherical mirror $\left(\rho^{\prime \Delta}\right)$.]

Les imperfections d'un tel système sont évidentes : d'abord les aberrations inhérentes à son principe même, puis la nécessité d'avoir un détecteur plus grand que l'échantillon pour recueillir tous les rayons partant de celui-ci, d'où une diminution du rapport signal sur bruit, le bruit étant proportionnel à la 
surface du détecteur. D'autre part, des réflexions multiples peuvent apparaître entre l'échantillon et le détecteur, augmentant ainsi artificiellement le facteur de réflexion de l'échantillon. De plus, la sensibilité du détecteur dépend de l'angle d'incidence des rayons, de sorte que tous les rayons réfléchis par l'échantillon ne sont pas détectés de la même manière. Pour ces différentes raisons, l'hémisphère de Coblentz mène à des déterminations peu précises. Sa réalisation est cependant assez facile et ce système peut servir pour des déterminations intégrées sur le spectre solaire [19] aussi bien que monochromatiques dans les domaines visible ou infra-rouge.

Pour éliminer les aberrations et utiliser le détecteur sous des incidences plus réduites, Blevin et Brown [20] ont utilisé un miroir ellipsoïdal : l'échantillon est placé à l'un des foyers et le détecteur à l'autre (Fig. 8). Plus performant que le précédent, ce système nécessite toujours un détecteur de surface importante. En effet, la réduction du cône d'incidence sur le détecteur conduit à placer ce dernier assez loin de l'ellipsoïde, de sorte que l'image de l'échantillon est de grande taille.

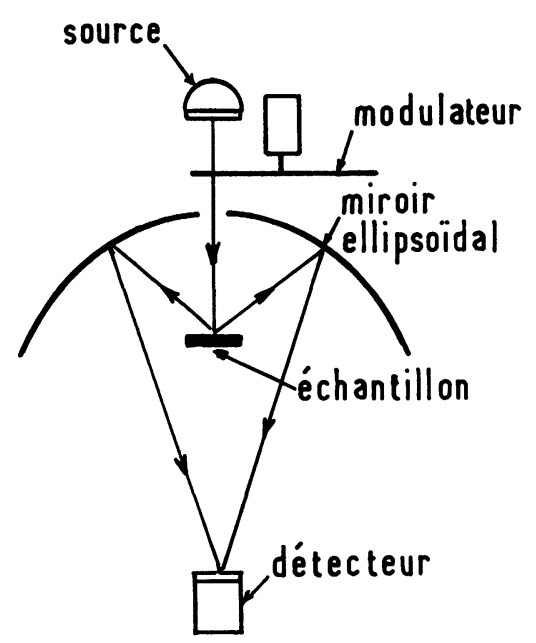

Fig. 8. - Miroir ellipsoïdal de Blevin $\left(\rho^{\circ}\right)$.

[Blevin's ellipsoïdal mirror $\left(\rho^{\prime \bullet}\right)$.]

Au lieu de mesurer $\rho^{\prime \Delta}$, d'autres montages fournissent $\rho^{\sigma^{\prime}}$ : ils utilisent des systèmes de miroirs, mais s'apparentent également aux sphères intégrantes que nous décrirons plus loin. C'est ainsi que :

- Janssen et Torborg [21] ont réalisé, selon la longueur d'onde choisie, différents diffuseurs grâce auxquels le flux incident est réfléchi de façon isotrope vers un miroir sphérique; celui-ci réfléchit spéculairement ce flux sur l'échantillon qui est ainsi éclairé de façon isotrope. Le flux réfléchi par l'échantillon dans la direction normale est alors mesuré après avoir traversé le miroir sphérique par une fenêtre symétrique de celle traversée par le flux incident (Fig. 9). La difficulté réside dans la réalisation d'un diffuseur parfait, ce qui est malaisé au-delà de $2 \mu \mathrm{m}$.

- White [22] remplace le diffuseur (Fig. 10) par

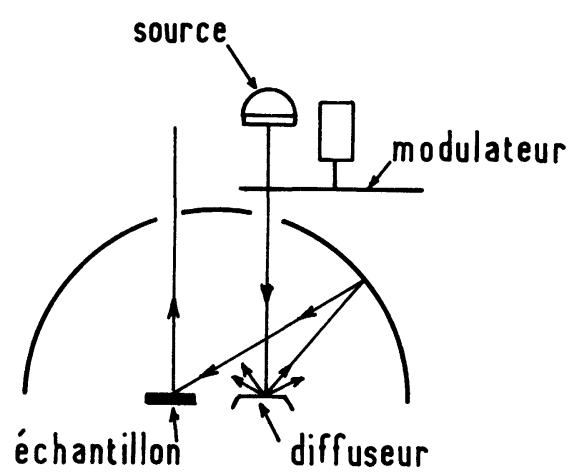

Fig. 9. - Miroir hémisphérique fonctionnant en sphère intégrante, de Janssen et Torborg $\left(\rho^{\circ}\right)$.

[Janssen and Torborg's hemispherical mirror functioning as an integrating sphere $\left(\rho^{\infty}\right)$.]

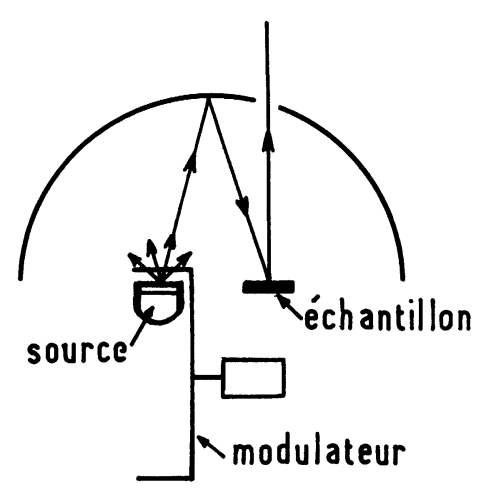

Fig. 10. - Miroir hémisphérique fonctionnant en sphère intégrante, de White $\left(\rho^{\triangle !}\right)$.

[White's hemispherical mirror, functioning as an integrating sphere $\left(\rho^{{ }^{\prime}}\right)$.]

une source infrarouge en nichrome dépoli, supposée isotrope. Il conserve la possibilité de moduler la source, afin de permettre le chauffage de l'échantillon sans pour autant perturber la mesure par l'émission propre de l'échantillon. Cependant, l'anisotropie de l'éclairement de l'échantillon reste le principal inconvénient de cette méthode.

- Plus récemment, Neher et Edwards [23] ont réalisé un réflectomètre pour l'infrarouge lointain $(\lambda=30$ à $100 \mu \mathrm{m})$ qui, contrairement aux deux montages précédents, subordonnés à une grande ouverture des miroirs sphériques, élimine les aberrations grâce à l'emploi de miroirs paraboliques(Fig. 11). Ce perfectionnement de la sphère de Coblentz permet des mesures précises, mais au prix d'une complication et donc d'un coût de réalisation non négligeable.

On notera qu'un autre inconvénient, commun à tous les systèmes précédents, est de ne permettre les mesures que selon une seule direction d'incidence ou de réflexion.

6.2 UTILISATION DE SPHÈRES INTÉGRANTES. - Les sphères ou demi-sphères dites « intégrantes " sont des 


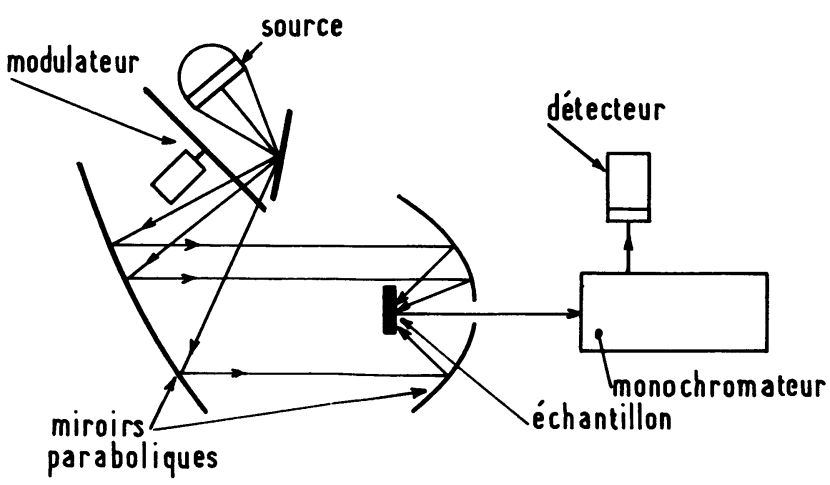

Fig. 11. - Système de miroirs paraboliques utilisé par Neher et Edwards pour l'obtention de $\rho^{\circ \prime}$.

[Parabolic mirrors system as used by Neher and Edwards to obtain $\rho^{\Omega^{\prime}}$.]

réflecteurs diffusants permettant soit de recueillir sur un détecteur de rayonnement le flux hémisphérique réfléchi par un échantillon-plan, soit d'éclairer un échantillon avec un flux hémisphérique diffusisotrope (dans la mesure du possible).

Les sphères ou demi-sphères intégrantes ont été surtout utilisées pour la mesure de $\rho^{\Delta \prime}$. Plusieurs montages ont été proposés :

- dès 1928, McNicholas [24] réalisa une demisphère de verre dépoli éclairée par cent quatre lampes et un réflecteur couvert d'oxyde de magnésium(Fig. 12),

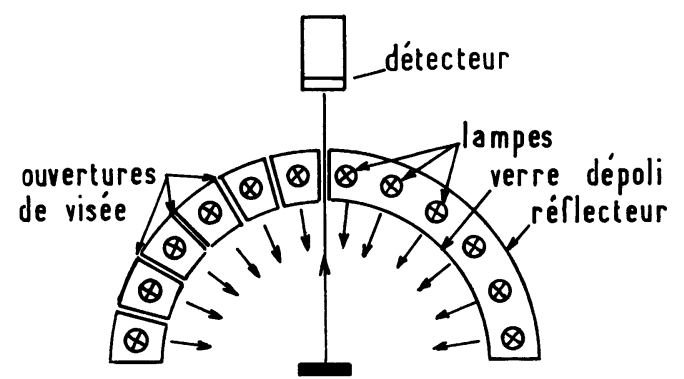

Fig. 12. - Sphère intégrante à lampes multiples, de Mc Nicholas $\left(\rho^{\iota^{\prime}}\right)$.

[McNicholas' integrating sphere (104 lamps, $\left.\rho^{{ }^{\prime}}\right)$.]

de manière à illuminer de façon isotrope un échantillon placé au centre de la demi-sphère. Différentes ouvertures pratiquées dans cette sphère permettaient de faire des mesures à plusieurs angles d'incidence;

- la disposition précédente, compliquée mais efficace, a été modifiée par Tingwaldt [25] qui n'utilisait plus qu'une seule lampe placée en haut d'une sphère recouverte de zinc blanc et supposée réfléchir de manière parfaitement diffuse le flux reçu de la lampe (Fig. 13). L'échantillon était placé au centre de la sphère sur un support tournant par rapport à l'ouverture par laquelle était effectuée la mesure du flux réfléchi par l'échantillon. Un écran était intercalé entre la lampe et l'échantillon, afin que celui-ci ne reçoive de flux que de la sphère. Ce système, beaucoup plus

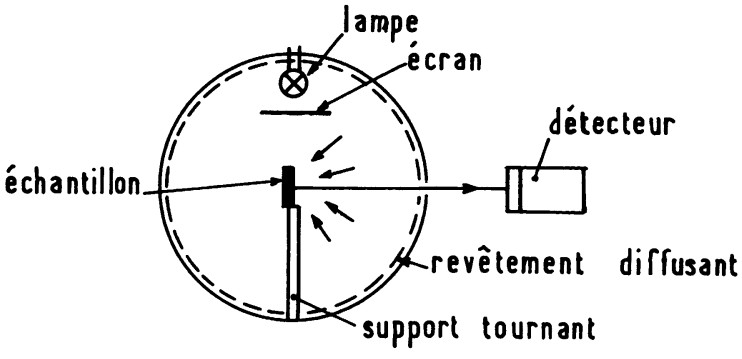

Fig. 13. - Sphère intégrante de Tingwaldt $\left(\rho^{{ }^{\prime}}\right)$. [Tingwaldt's integrating sphere $\left(\rho^{\Delta^{\prime}}\right)$.]

simple que celui de McNicholas, était cependant très imparfait par suite de l'éclairement non uniforme de la sphère par la lampe. En effet, la lampe aurait dû être au centre de la sphère ou sur la sphère [16] pour que son éclairement soit uniforme, ce qui assure l'isotropie de l'éclairement de l'échantillon, lorsque le revêtement de la sphère réfléchit de façon diffuse isotrope;

- dans cet ordre d'idées, Toporets [26] a réalisé, en 1959, une sphère identique à celle de Tingwaldt, la lampe étant remplacée par un verre diffusant éclairé extérieurement par une source (Fig. 14). Malheureusement, la difficulté de réalisation d'un verre à transmission parfaitement diffusante ne permet pas d'obtenir de très bons résultats.

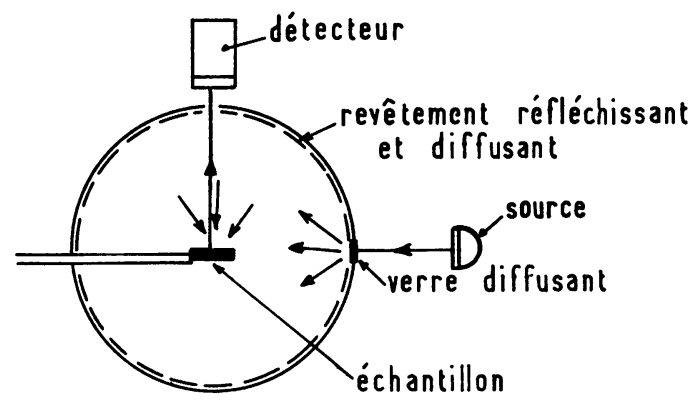

Fig. 14. - Sphère intégrante de Toporets $\left(\rho^{\triangle^{\prime}}\right)$.

[Toporets' integrating sphere $\left(\rho^{{ }^{\prime}}\right)$.]

Les sphères intégrantes sont toutes basées sur la réalisation d'un revêtement de paroi à la fois très réfléchissant, afin que la sphère ait un rendement correct et aussi très diffusant pour que les réflexions multiples assurent un éclairement uniforme de la sphère. Cette condition est facile à réaliser dans le domaine visible avec les revêtements classiques d'oxyde de magnésium ou, plus récemment, par l'emploi de diverses peintures commercialisées à cet effet [6]. Par contre, au-delà de $\lambda=2 \mu \mathrm{m}$, les revêtements sont beaucoup plus difficiles à trouver, ce qui explique que les sphères intégrantes ont longtemps été réservées à un usage dans le visible ou le très proche infrarouge. Des progrès récents ont été cependant obtenus par Imbert [27] dans le domaine des revêtements diffusants pour l'infrarouge. Il a réalisé une sphère inté- 
grante donnant des résultats satisfaisants pour la mesure de $\rho^{\Delta^{\prime}}$ : des petites billes de verre (diamètre $0,6 \mathrm{~mm}$ ) sont collées sur la face interne de la sphère, puis revêtues par vaporisation sous vide de deux couches successives de chrome et d'or. Dans ce montage (Fig. 15), la sphère est éclairée en principe uniformément par une source annulaire entourant l'échantillon et non visible de celui-ci; le flux réfléchi par

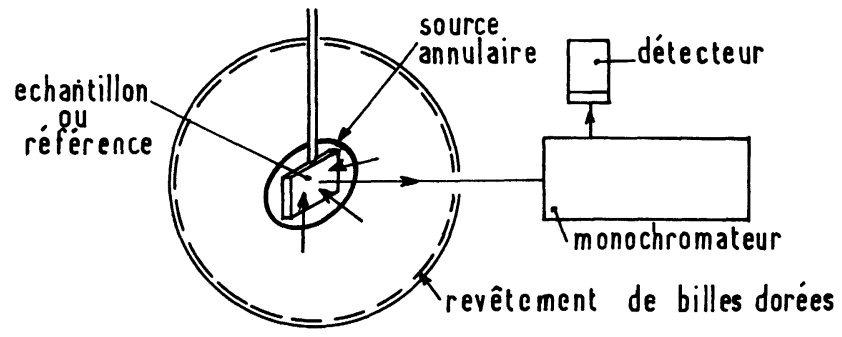

Fig. 15. - Sphère intégrante d'Imbert pour l'infrarouge $\left(\rho^{\circ \prime}\right)$.

[Imbert's integrating sphere (I.R. r gnge, $\left.\rho^{{ }^{\prime}}\right)$.]

l'échantillon dans la direction correspondant à l'orifice de la sphère est détecté après passage dans un monochromateur. Ce type de revêtement permet donc de généraliser l'usage des sphères intégrantés à l'infrarouge. Toutefois, ces montages ont un rendement très faible, ce qui implique l'emploi de sources puissantes, difficiles à réaliser dans l'infrarouge. C'est pourquoi, il y a peu de réalisations de ce type; signalons simplement celle de Godon [28] qui a repris le montage d'Imbert en plaçant la source à l'extérieur de la sphère, ce qui permet de la moduler et donc de faire des mesures sur un échantillon chauffé, puisque l'émission propre - non modulée - peut être éliminée par l'emploi d'une détection synchrone. Enfin, le même principe a été utilisé récemment dans le domaine visible par Behaghel [11], le revêtement de billes étant remplacé par une peinture blanche diffusante. Une étude récente [16] a montré cependant que la disposition de la source à l'extérieur de la sphère est beaucoup plus contraignante en ce qui concerne la qualité du revêtement diffusant pour l'infrarouge. Des travaux sont en cours sur ces questions dans les laboratoires des auteurs.

Les mesures de $\rho^{\prime}$ peuvent également être obtenues à l'aide de sphères intégrantes ; ainsi, Edwards et al. [29] ont mis au point, en 1960, une sphère revêtue d'oxyde de magnésium pour faire des mesures jusqu'à $2 \mu \mathrm{m}$. Une source monochromatique (Fig. 16) éclaire un échantillon placé sur un support tournant et réfléchissant la lumière vers la sphère. Un détecteur est disposé sous l'échantillon de manière à ce que le flux directement réfléchi par l'échantillon sur le détecteưr soit négligeable. On retrouve ici l'inconvénient signalé pour l'hémisphère de Coblentz, à savoir la nécessité d'utiliser un détecteur ayant une réponse angulaire uniforme, afin que celui-ci "voie" toute la sphère

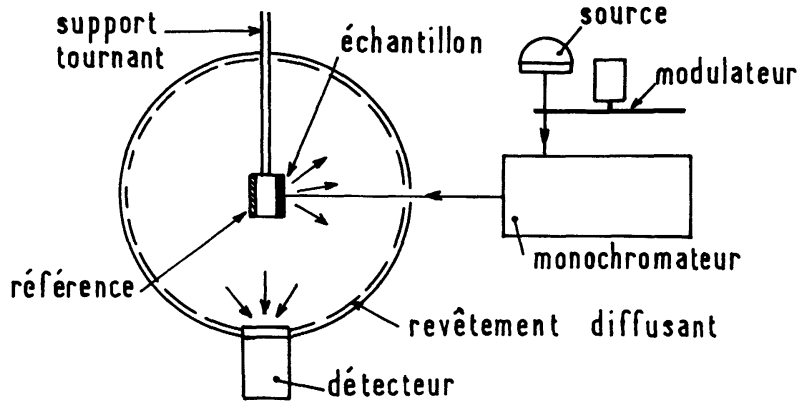

Fig. 16. - Sphère intégrante d'Edwards et al. $\left(\rho^{\prime \bullet}\right)$.

[Edwards et al. integrating sphere $\left(\rho^{{ }^{\prime}}\right)$.]

avec la même sensibilité, ce qui n'est pas réalisable parfaitement. De plus, l'échantillon et son support masquent une partie non négligeable de la sphère.

Les montages décrits précédemment sont le plus souvent utilisés pour des mesures relatives, en opérant d'abord sur une référence, puis sur l'échantillon. Cependant, contrairement aux systèmes à miroirs, les sphères intégrantes permettent en général de réaliser des mesures absolues par comparaison avec une référence prise sur la paroi de la sphère : dans les déterminations de $\rho^{\circ}$, avant d'effectuer la mesure sur l'échantillon, on procède à une mesure sur le revêtement de la sphère, alors que dans les montages déterminant $\rho^{\prime}$ la source est d'abord dirigée sur le revêtement, ce qui fournit une référence, avant d'être dirigée vers l'échantillon.

Outre la nécessité d'un revêtement réfléchissant de façon diffuse-isotrope, sans lequel les mesures seraient erronées, deux autres précautions doivent également être prises : dans la mesure de $\rho^{\Delta \prime}$, l'échantillon ne doit pas « voir » la source; dans celle de $\rho^{\prime \triangle}$, le détecteur ne doit pas « voir » l'échantillon.

Dans l'article suivant, les auteurs donneront la description des montages à sphères intégrantes réalisés dans leurs laboratoires.

6.3 Utilisation Des Cavités Chauffées. - Dans les années 1950, est apparue une nouvelle méthode de mesure pour le domaine infrarouge, proposée par Gier et al. [30]. L'échantillon est placé dans une cavité chauffée uniformément à une température d'environ $1000 \mathrm{~K}$ (Fig. 17); une fenêtre permet de mesurer le flux réfléchi dans cette direction par l'échantillon.

Des mesures directionnelles sont possibles si l'échantillon est monté sur un support tournant [31]. Ce système est cependant de réalisation assez délicate. En effet :

- des gradients de température même faibles, difficiles à éviter, peuvent produire des erreurs sensibles, surtout lorsque l'appareil est utilisé sans référence; une mesure est alors effectuée sur la paroi de la cavité, puis sur l'échantillon;

- la modulation du flux incident n'étant pas possible, on ne peut pas séparer l'émission propre de l'échantillon du flux réfléchi par celui-ci, ce qui limite 


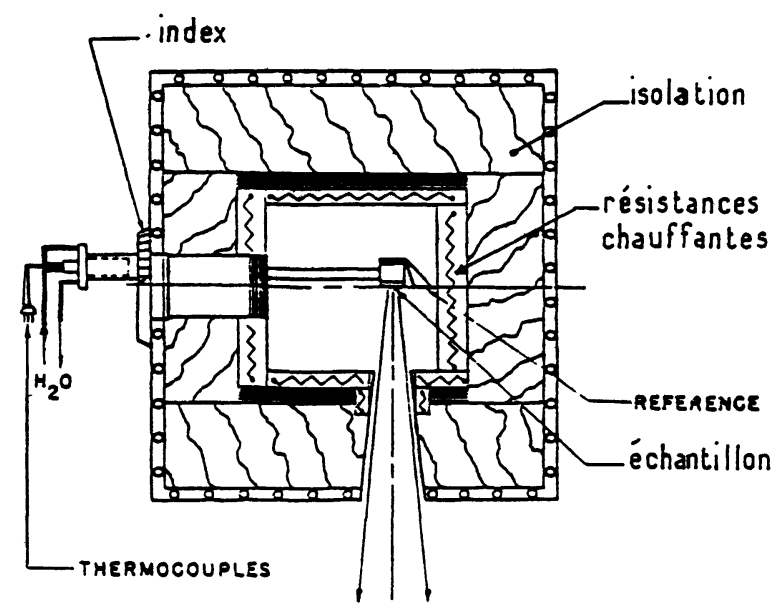

Fig. 17. - Réflectomètre à cavité chauffée pour la mesure de $\rho^{\circ \prime} M$ résistance chauffante; 0000 refroidissement par circulation d'eau; $\equiv$ écran antirayonnant; isolant thermique.

[Heated cavity reflectometer for $\sigma^{\circ \prime}$ measurement : MM heating resistor; 0000 cooling water circuit; antiradiating screen; thermal insulation.]

la gamme de longueur d'onde de plus en plus au fur et à mesure que la température de l'échantillon s'élève. En effet, la luminance monochromatique d'un corps noir à $300 \mathrm{~K}$ représente moins de $0,1 \%$ de celle d'un corps noir à $1000 \mathrm{~K}$ jusqu'à environ $5 \mu \mathrm{m}$; par contre, ce rapport passe à $10 \%$ à $20 \mu \mathrm{m}$. Pour un corps noir à $700 \mathrm{~K}$, ces mêmes rapports deviennent respectivement $27 \%$ et $57 \%$. Si l'on souhaite explorer un domaine relativement étendu de longueur d'onde, il est clair que les mesures ne peuvent être faites qu'à des températures voisines de l'ambiante; l'échantillon doit donc être vigoureusement refroidi puisqu'il est placé dans une cavité à $1000 \mathrm{~K}$. Cette nécessité limite l'usage de la méthode à des corps bons conducteurs de la chaleur;

- de plus, les parois métalliques de la cavité risquent d'introduire des erreurs supplémentaires si certaines précautions ne sont pas prises [31]. En effet, des réflexions multiples peuvent apparaître entre l'échantillon et les parois de la cavité; par ailleurs, l'échantillon est susceptible de "voir" par réflexion sur la paroi une image de la fenêtre de mesure. Il faut donc utiliser des parois à réflexion faible et aussi peu spéculaires que possible et, par exemple, usiner des macrorugosités sur celles-ci.

7. Mesure des grandeurs directionnelles intégrées sur une large bande de longueurs d'onde. - Dans certaines applications, on s'intéresse à des grandeurs directionnelles intégrées sur une large bande de longueurs d'onde $\left[\lambda_{1}, \lambda_{2}\right]$, le plus souvent $\varepsilon_{\lambda_{1} \lambda_{2}}^{\prime}$ ou $\alpha_{\lambda_{1} \lambda_{2}}^{\prime}$. Les limites de cette bande peuvent être celles du phénomène d'émission lui-même, auquel cas il s'agit de grandeurs directionnelles totales, par exemple $\alpha_{\mathrm{s}}^{\prime}$ l'absorptivité directionnelle d'une surface pour le rayonnement solaire direct. Mais, $\lambda_{1}$ et $\lambda_{2}$ peuvent être également les limites de la bande passante du système récepteur, comme c'est le cas par exemple dans les mesures d'émissivité réalisées avec une caméra infrarouge (cf. Martin et Fauchais [32]).

La plupart des dispositifs destinés aux mesures directionnelles monochromatiques décrits dans ce qui précède peuvent être, dans leur principe, étendus à la mesure des grandeurs intégrées sur une bande spectrale plus ou moins large. Il suffit de remplacer le monochromateur par un système optique ayant la bande passante désirée.

Si l'on recherche en particulier des grandeurs totales, seuls les détecteurs du type thermique (thermoélectriques, pyroélectriques ou pneumatiques) peuvent convenir et encore faut-il veiller soigneusement au choix de leur fenêtre, voire la supprimer dans certains cas.

8. Mesure des grandeurs hémisphériques totales : les méthodes calorimétriques. - Bien que le calcul précis des échanges radiatifs entre divers éléments nécessite en général la connaissance de facteurs monochromatiques directionnels, dans certaines applications, on peut se satisfaire de grandeurs moyennées. Parmi ces dernières, les plus employées sont l'émissivité totale hémisphérique $\varepsilon^{\circ}$ et l'absorptivité solaire directionnelle $\alpha_{s}^{\prime}(\theta)$. En ce qui concerne ce dernier paramètre, il s'agit le plus souvent de mesurer $\alpha_{s}^{\prime}(0)$, dans la direction normale à la surface. Ces facteurs interviennent dans la détermination de l'équilibre thermique d'un véhicule spatial ou dans les calculs simplifiés du comportement des capteurs solaires. Les méthodes calorimétriques sont les seules qui permettent d'atteindre $\varepsilon^{\circ}$. Elles fournissent en outre directement $\alpha_{\mathrm{s}}^{\mathrm{A}}$ ou $\alpha_{\mathrm{s}}^{\prime}$, c'est-à-dire sans passer par l'intermédiaire de mesures de réflectivité.

8.1 Mesure De l'absorptivité SOlaire $\alpha_{s}$. - Une méthode proposée par Birkebak et Hartnett [33] consiste à exposer au soleil un échantillon et une référence d'absorptivité connue. Ces deux éléments, isolés sur leur face arrière, sont placés dans un environnement à température constante (Fig. 18). Lorsqu'un

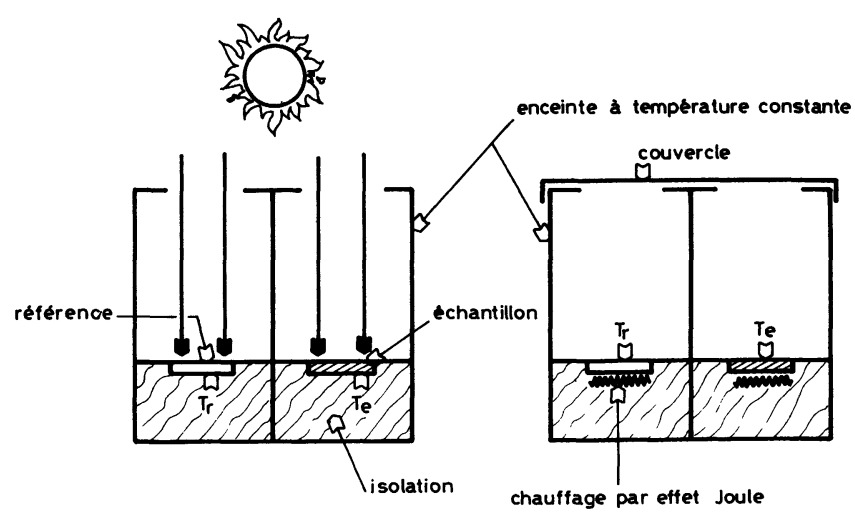

Fig. 18. - Principe d'une mesure calorimétrique de $\alpha_{\mathrm{s}}$. [Calorimetric measurement of $\alpha_{s}$.] 
écran intercepte les rayons solaires, on compense l'apport du soleil par un chauffage électrique des deux éléments de manière à ce que leur température reste la même que lorsque le soleil les chauffait. Le rapport des puissances fournies par les résistances électriques à l'échantillon, d'une part, et à la référence, d'autre part, est alors égal au rapport de leurs absorptivités solaires $\alpha_{s}$. Cette méthode simple est cependant peu précise par suite de la nécessité de maintenir des conditions d'éclairement solaire constantes pendant le temps nécessaire à l'établissement de l'équilibre thermique et aussi par suite du changement apporté à «l'environnement " lors des deux phases de la mesure.

\subsection{MESURE DE L'ÉMISSIVITÉ TOTALE HÉMISPHÉRIQUE.} - La méthode la plus courante consiste à chauffer par effet Joule un échantillon suspendu par les fils d'amenée de courant au milieu d'une enceinte noire refroidie [34]. La détermination de la température d'équilibre et de la puissance dissipée par effet Joule permet de déduire $\varepsilon^{\infty}$. Il est également possible de faire une mesure en transitoire à partir de la vitesse de refroidissement d'un échantillon dans une telle enceinte (Fig. 19). Mais, il est alors nécessaire de connaître la chaleur massique du matériau étudié.

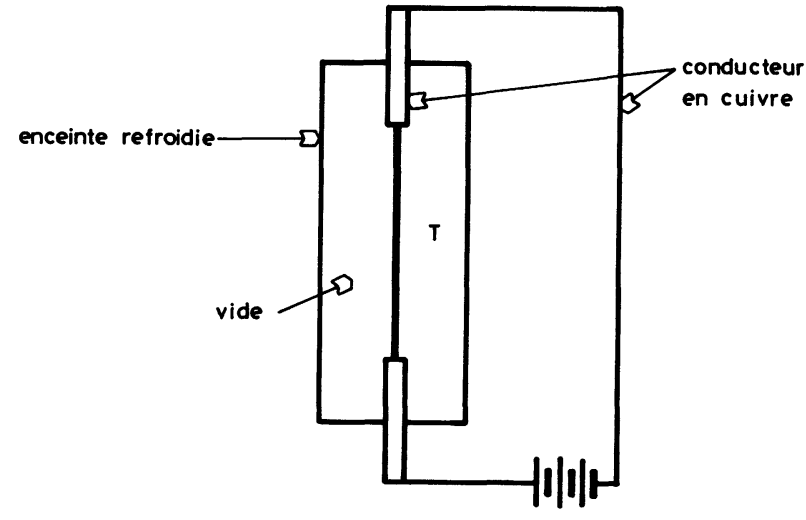

Fig. 19. - Principe de mesure de $\varepsilon^{\curvearrowright}$ par calorimétrie.

[Calorimetric measurement of $\varepsilon^{\circ}$.]

Dans le cas d'échantillons mauvais conducteurs électriques, une petite couche du matériau testé est appliquée sur un élément chauffant. La difficulté réside alors dans la mesure précise de la température de surface. Les méthodes de ce type ont fait l'objet d'une description détaillée par Gaumer et al. [35]. On trouvera également dans la référence [36] la description d'un montage récent, du type calorimétrique, réalisé au C.E.N. de Grenoble par Aubert.

Notons qu'en utilisant un chauffage solaire, la connaissance de la température d'équilibre suffit pour déterminer directement le rapport $\alpha_{S} / \varepsilon^{\circ}$, puisqu'il y a égalité des flux émis et absorbé.

Signalons enfin une méthode utilisant un flux incident modulé permettant de calculer $\varepsilon$ à partir du déphasage entre cette modulation du flux et la température de l'échantillon [37]. En effet, le calcul de celle-ci montre que le déphasage ne dépend que de $\varepsilon^{\circ}$, des propriétés physiques de l'échantillon (masse, capacité calorifique), qu'il faut par ailleurs connaître, et de la fréquence de modulation. Cette méthode permet également de calculer $\alpha_{S}$ ou $\alpha_{S} / \varepsilon^{\circ}$, si la source utilisée reproduit le spectre solaire et si une mesure de l'amplitude de la modulation peut être effectuée.

9. Conclusion. - Les principales méthodes de détermination des caractéristiques radiatives des matériaux opaques ont fait l'objet d'un examen critique et comparatif. La démarche adoptée a commencé par la présentation des techniques de mesure de la réflectivité monochromatique bidirectionnelle, que l'on peut considérer à juste titre comme le plus fin de tous les facteurs radiatifs. Elle s'achève par la description de méthodes de détermination des paramètres les plus globaux, à savoir les facteurs totaux hémisphériques, sans oublier les techniques de mesure des divers facteurs intégrés dans les domaines de l'espace ou de la longueur d'onde.

La réflectivité monochromatique bidirectionnelle permet, théoriquement du moins, d'atteindre tous les autres facteurs, mais sa mesure demeure, comme on l'a $\mathrm{vu}$, l'une des plus délicates en raison de la faiblesse des flux réfléchis, surtout dans le cas des matériaux diffusants.

Aussi, la plupart des autres techniques d'obtention, par voie directe ou indirecte, des réflectivités, émissivités et absorptivités monochromatiques directionnelles, décrites dans ce qui précède, conserveront encore longtemps tout leur intérêt.

Le problème important - trop souvent négligé dans un passé encore récent - des erreurs expérimentales introduites par les phénomènes de polarisation a été examiné et une solution simple a été proposée.

Une description détaillée des montages mis au point dans les laboratoires des auteurs et de certains progrès récents obtenus en métrologie des propriétés radiatives fait l'objet du prochain article de cette revue.

Remerciements. - Les auteurs remercient tout particulièrement MM. B. Heinisch et P. Quinto-Diez, chercheurs au Laboratoire d'Hydraulique et Dynamique des Gaz de l'I.N.S.A., pour leur précieuse collaboration. 


\section{Bibliographie}

[1] Siegel, R., Howell, J. R., Thermal Radiation Heat Transfer (McGraw-Hill, N.Y.) 1972.

[2] Sacadura, J. F., Application des techniques de MonteCarlo à l'évaluation des transferts radiatifs, Ecole d'été G.U.T.-C.N.R.S., Perpignan, septembre (1976) et cours D.E.A. Thermique, Ecole Centrale de Paris (1979).

[3] Tran Nguyen, H., Thèse Dr. Ing., Ecole Centrale Paris, juin (1981).

[4] Touloukian, Y. S., Dewit, D. P., Thermal Radiative Properties of Materials (Plenum Press, N.Y.) 1970, vol. 7, 8.

[5] Huetz-Aubert, M., TAIne, J., Rev. Gén. Thermique, tome XVII, 202 (1978) 755.

[6] Huetz-Aubert, M., Sacadura, J. F., Revue Phys. Appl., article suivant.

[7] Sacadura, J. F., Thèse d'Etat, No I.DE.8007, Lyon, juillet (1980).

[8] Sacadura, J. F., Quinto-Diez, P., Heinisch, B., VIIe Congrès International sur les transferts de chaleur, Munich (1982).

[9] Torrance, K. E., Sparrow, E. M., Trans. A.S.M.E., J. Heat Transfer, May (1965), 283-292.

[10] BirkebaK, R. C., ECKert, E. R. G., Trans. A.S.M.E., J. Heat Transfer 87 (1965) 85-94.

[11] Behaghel, J. M., Thèse Dr. Ingénieur, Paris VI (1980).

[12] Lafait, J. et al., J. Physique Colloq. 42 (1981) Cl.

[13] Abukadir, A., Spectral Directional Emittance of Roughened Metal Surfaces, Ph. D, Univ. Kentucky, № 73-29884 (1973).

[14] Hsia, J. J. Richmond, J. C., J. Res. Nat. Bur. Stand. 80A (1976) 189-205.

[15] Hervé, Ph., Thèse d'Etat, Paris VI (1977).

[16] Demont, Ph., Thèse Dr. Ingénieur, Ecole Centrale des Arts et Manufactures, Paris (1980).

[17] Sacadura, J. F., Rev. Int. Hautes Temp. et Réfract. 8 (1971) 101-110.

[18] Coblentz, W. W., The Diffuse Reflecting Power of Various Substances, Bull. Nat. Bur. Std., 9 (1913), 283-325, cité par Dunn, Richmond, Parmer. Journal of Spacecraft and Rockets, vol. 3, $\mathrm{n}^{\circ}$ 7, July (1966) 961-975.

[19] Birkebak, R. C., Hartnett, J. P., Eckert, E. R. G., Progress in Int. Res. Thermodynamics and Transport Properties, A.S.M.E. (1962) 563-574.
[20] Blevin, W. R., Brown, W. J., An Infrared Reflectometer with a Spheroidal Mirror, J. Sci. Instrum. 42 (1965) 385-389.

[21] JAnssen, J. E., Torborg, R. M., in Measurement of Thermal Radiation Properties of Solids, Edit. Richmond, NASA SP-31 (1963) 169-182.

[22] White, J. U., J. Opt. Soc. Am. 54 (1965) 1332-1337.

[23] Neher, R. T., Edwards, D. K., Appl. Opt. 4 (1965) 775780.

[24] McNicholas, H. J., J. Res. Nat. Bur. Stand. 1 (1928) 29-73.

[25] TingwaldT, C. P., Optik 9 (1952) 323-332.

[26] Toporets, A. S., Opt. Spectrosc. 7 (1959) 471-473.

[27] IMBERT, C., Thèse Dr. Ingénieur, Paris XI (1969).

[28] Godon, J. C., Thèse de 3 e cycle, Paris VI (1977).

[29] Edwards, D. K., Gier, J. T., Nelson, K. E., RodDiCK, R. D., Appl. Opt. 51 (1961) 1279-1288.

[30] Gier, J. T., Dunkle, R. V., Bevans, J. T., J. Opt. Soc. Am. 44 (1954) 558-565.

[31] Dunkle, R. V., Edwards, D. K., Gier, J. T., Nelson, K. E., RodDICK, R. E., in Progress in Intern. Res. Thermodynamics and Transport Properties, A.S.M.E. New York (1962) 541-562.

[32] Martin, C., Fauchais, P., Revue Phys. Appl. 15 (1980) 1469-1478.

[33] Birkebak, R. C., Hartnett, J. P., Trans. A.S.M.E., J. Heat Transfer 80 (1958) 373-378.

[34] Henninger, L., Méthode de mesure de l'émittance hémisphérique d'un revêtement, Note technique du C.N.E.S., $\mathrm{n}^{\mathrm{o}}$ 53, octobre (1976).

[35] Gaumer, R. E., McKellar, L. A., Streed, E. R., Frame, K. L., Grammer, J. R., in Calorimetric Determinations of Thermal Radiation Characteristics, Progress in Int. Res. Thermodynamic and Transfer Properties (1962) 575-587.

[36] Aubert, A., J. Physique Colloq. 42 (1981) C1-383-387.

[37] JACK, J., A.I.A.A. J. 5 (1967) 1603-1606.

\section{Références générales}

- Measurement of Thermal Radiation Properties of Solids, Symp. of Dayton, Ohio, September (1962), ed. by Joseph C. Richmond, NASA SP 31 .

- Symposium on Thermal Radiation of Solids, SanFrancisco (1964), ed. by S. Katzoff, NASA SP 55. 